\title{
The preparation, characterization and biological activity of palladium(II) and platinum(II) complexes of tridentate NNS ligands derived from S-methyl- and S- benzyldithiocarbazates and the $\mathrm{X}$-ray crystal structure of the $[\mathrm{Pd}(\mathrm{mpasme}) \mathrm{Cl}]$ complex.
}

\begin{abstract}
New complexes of general formula, [M(NNS)Cl] $(\mathrm{M}=$ PdII, PtII; NNS = anionic forms of the 6-methyl-2-formylpyridine Schiff bases of S-methyl- and S-benzyldithiocarbazates) have been prepared and characterized by a variety of physico-chemical techniques. Based on conductance and spectral evidence, a square-planar structure is assigned to these complexes. The crystal and molecular structure of the $[\mathrm{Pd}(\mathrm{mpasme}) \mathrm{Cl}]$ complex (mpasme=anionic form of the 6-methyl-2-formylpyridine Schiff base of S-methyldithiocarbazate) has been determined by X-ray diffraction. The complex has a distorted square-planar geometry with the ligand coordinated to the palladium(II) ion via the pyridine nitrogen atom, the azomethine nitrogen atom and the thiolate sulfur atom; the fourth coordination position around the palladium(II) ion is occupied by the chloride ligand. The distortion from a regular squareplanar geometry is ascribed to the restricted bite angle of the ligand. Both the Schiff bases exhibit strong cytotoxicity against the human ovarian cancer (Caov-3) cell lines, the S-methyl derivative being two times more active than the S-benzyl derivative. The [Pt(mpasme)Cl] complex is moderately active but the palladium(II) complex is weakly active against this cancer. None of the complexes of Hmpsbz are active against Caov-3. The Schiff base, Hmpasme exhibits moderate activity against the bacteria, MRSA, P. aeruginosa and S. typhimurium but is inactive against B. subtilis. Coordination of the ligand with palladium(II) substantially reduces its activity. The Schiff base, Hmpasbz and its palladium(II) and platinum(II) complexes are inactive against these bacteria. The Schiff bases and their palladium(II) and platinum (II) complexes are inactive against the pathogenic fungi, C. albican, Aspergillus ochraceous and Saccharomyces cerevisiae.
\end{abstract}

Keyword: Dithiocarbazate; Crystal structure; Pd(II); Pt(II); 6-methyl-2- formylpyridine schiff base. 